\title{
Asymptomatic SARS-CoV-2 Carriers may not Generate Antibodies: A Case
}

\section{Report}

\section{Yushuai Liu | Fagang Jiang*}

\section{*Correspondence: Fagang Jiang}

Address: Department of Ophthalmology, Union Hospital, Tongji Medical College, Huazhong University of Science and Technology, Wuhan 430022, China

e-mail $\bowtie:$ 13554100999@163.com

Received: 13 November 2020; Accepted: 19 November 2020

Copyright: (C) 2020 Liu Y. This is an open-access article distributed under the terms of the Creative Commons Attribution License, which permits unrestricted use, distribution, and reproduction in any medium, provided that the original work is properly cited.

\section{ABSTRACT}

Asymptomatic SARS-CoV-2 carriers are infectious to some extent and can potentially transmit coronavirus disease 2019 (COVID-19). RNA assay combining antibody detection significantly improved the sensitivity of pathogenic diagnosis for COVID-19. Here, we report an asymptomatic case of COVID-19 with positive RNA but consistently negative antibodies (IgM and IgG). This case indicates that not everyone can generate specific antibodies after infection with SARS-CoV-2. The absence of antibodies means that the asymptomatic carriers like our case have no specific resistance to the virus and their plasm may do no good in the treatment of the other COVID-19 patients. As RNA detectability of COVID-19 patients is not too high and will further decrease with the duration of disease, negative antibodies results may miss diagnosis. Therefore, repeated nucleic acid assay and antibody test should be carried out for suspected cases.

Keywords: COVID-19, SARS-CoV-2, Asymptomatic Carrier, Antibody

\section{Introduction}

A novel coronavirus (SARS-CoV-2) disease (COVID-19) was first identified after an outbreak in Wuhan, Hubei Province, China (Zhu et al., 2020). China has now transitioned to the mitigation stage, but it still faces the dual risk of sporadic new cases and imported cases (Zhang et al., 2020a). However, asymptomatic carriers are "silent spreaders" and also warrant attention in terms of disease prevention and epidemic containment. A growing number of studies has indicated that asymptomatic carriers are infectious to some extent and can potentially transmit COVID-19 (Rothe et al., 2020; Bai et al., 2020; Wang et al., 2020). Accurate and fast diagnosis of the causative SARS-CoV-2 is crucial in terms of the patient isolation and epidemic containment. RNA assay combining antibody detection significantly improved the sensitivity of pathogenic diagnosis for COVID-19 (Zhao et al., 2019; Xiang et al., 2020). 
Here, we report an asymptomatic case of COVID-19 with positive RNA but consistently negative antibodies.

\section{Case Presentation}

A 46-year-old man without any obvious symptoms was asked for disease screening at the fever clinic of Union Hospital of Tongji Medical College, Huazhong University of Science and Technology on February 7, 2020, due to his close contact with a confirmed COVID-19 patient (Fig. 1). A high-resolution computed tomography (HRCT) of the chests was performed, images of which reported no obvious disorders. Blood routine and C-reactive protein were also normal. On February 8, the man's oropharyngeal swab test of SARS-CoV-2 by qualitative real-time reverse-transcriptase-polymerasechain-reaction (RT-PCR) assay was positive but negative at a second consecutive test on the following day. The man was put into quarantine at a hotel for medical observation. On March 6, SARS-CoV-2 antibodies (IgM and IgG) were tested and were negative. However, the result of the oropharyngeal swab test of SARS-CoV-2 RNA was still positive on March 8. The man was then admitted to hospital for further examination and isolation. Oropharyngeal swab tests of SARS-CoV-2 RNA were performed repeatedly for surveillance on March 11, March 12, and March 14, and all were negative. Laboratory examinations for blood routine, liver function, renal function, electrolyte levels, blood coagulation function, serum procalcitonin, C-reactive protein, and IL-6 were normal. The HRCT of the chests still displayed no lesion on March 13. The antibodies of SARS-CoV-2 were still negative on March 14. The man was then transferred to the quarantined hotel for more observation on March 17. After fourteen days of this observation, the retests of SARS-CoV-2 RNA and antibodies were negative and the man was released from quarantine on March 31. In all the observation period, he displayed no fever or any other symptoms.

\begin{tabular}{|c|c|c|c|c|c|c|c|c|c|c|}
\hline & & PCR(+) & PCR(-) & & PCR(+) & PCR(-) & PCR(-) & & PCR(-) & PCR(-) \\
\hline C & CT(-) & & & $A b(-)$ & & & & CT(-) & $A b(-)$ & $A b(-)$ \\
\hline & $7-\mathrm{Feb}$ & 8-Feb & 9-Feb & 6-Mar & 8-Mar & 11-Mar & 12-Mar & 13-Mar & 14-Mar & 31-Mar \\
\hline
\end{tabular}

Figure 1: Timeline of the asymptomatic SARS-CoV-2 carrier

C: contact history with a confirmed COVID-19 patient; CT: high-resolution computed tomography of the chests; PCR: oropharyngeal swab test of SARS-CoV-2 by qualitative real-time reverse-transcriptase-polymerase-chain-reaction assay; Ab: SARS-CoV-2 antibodies (IgM and IgG); +: positive; -: negative.

\section{Discussion}

Asymptomatic infection was recognized as a source of infection by the Diagnosis and Treatment Protocol for COVID-19 (Fifth ed., Trial) on February 5, 2020 (the National Health Commission of the 
People's Republic of China). Accurate and rapid diagnosis of the causative SARS-CoV-2 is important in order to isolate COVID-19 patients timely and helps stop the epidemic. The nucleic acid tests or genetic sequencings serve as the gold standard method for the confirmation of infection, but have frequent false-negative results because of sampling or potential absence of viruses in the respiratory system (Zhao et al., 2019; Xiang et al., 2020; Corman et al., 2020). Combining antibody detection can significantly improve the sensitivity of pathogenic diagnosis (Zhao et al., 2019; Xiang et al., 2020).

The seroconversion of specific IgM and IgG antibodies can be detected as early as on the 4th day after symptom onset, and generally IgM cumulative seroconversion increased from the 9th day, while IgG the 11th day (Xiang et al., 2020). Both antibodies were seropositive in nearly all the patients within the illness course more than 30 days (Xiang et al., 2020). Another study found that on the 19th day after symptom onset, $100 \%$ of patients tested positive for antiviral IgG (Long et al., 2020). Different from the reported literatures, the antibody tests in this case were all negative, even to the 52 th day after the first finding of SARS-CoV-2 RNA. As we know, RT-PCR has been widely deployed in diagnostic virology and has yielded few false-positive outcomes (Corman et al., 2020). This case had twice positive results of SARS-CoV-2 RNA, and was indeed an asymptomatic carrier. The negative result of SARS-CoV-2 RNA on February 9 may have been a false negative. One study in our hospital found that in the confirmed patients with COVID-19, the sensitivity, specificity, positive predictive value, negative predictive value, and consistency rate of IgM were $77.3 \%, 100 \%, 100 \%, 80.0 \%$, and $88.1 \%$, and those of IgG $83.3 \%$, 95.0\%, 94.8\%, 83.8\%, and 88.9\%, respectively (Xiang et al., 2020). Given this, we affirm that asymptomatic carriers may not generate antibodies in some cases.

A recent study found that immediate antibody responses were identified among severe cases compared to non-severe cases (Yongchen et al., 2020). Moreover, a higher titer of antibody was independently associated with a worse clinical classification (Zhao et al., 2019). Similarly, a previous study of severe acute respiratory syndrome coronavirus found that deceased infected patients reached the peak of anti-spike neutralizing antibody much earlier than clinical recovered patients (Zhang et al., 2006). High viral load can drive strong extrafollicular B cell responses, leading to rapid antibody responses (Lam and Baumgarth, 2019; Baumgarth, 2013). Asymptomatic carriers may have low viral load or strong innate immunity so that the virus may be cleared by innate immunity alone. This may explain the negative antibodies in our case. Of course, since the sensitivity of both IgM and IgG is not $100 \%$, the possibility of false negatives of the antibodies existed.

This case indicated that not everyone can generate specific antibodies after infection with SARSCoV-2. The absence of antibodies means that the asymptomatic carrier had no specific resistance to the virus. The infected cases who do not generate antibodies have the possibility of being re-infected. 
Plasma from patients recovered from COVID-19 that contains antibodies has shown promising efforts on patients with severe COVID-19 (Duan et al., 2020; Zhang et al., 2020b). However, the plasma from asymptomatic carrier without specific antibodies like our case may do no good in the treatment of the other COVID-19 patients. As RNA detectability of COVID-19 patients is not too high and will further decrease with the duration of disease (Zhao et al., 2020), negative antibodies results may miss diagnosis. Therefore, repeated nucleic acid assay and antibody test should be carried out for suspected cases. Considering that this is only one case, further investigation is needed to confirm our finding.

\section{Declarations}

Conflict of Interest: All authors declare that they have no conflict of interest.

Human and Animal Rights and Informed Consent: This article does not contain any studies with human or animal subjects performed by any of the authors.

Ethics Statement: The patient described herein provided full informed consent for the report.

Authors' Contributions: Yushuai Liu contributed to data collection, analysis, and was primarily responsible for writing the text; Fagang Jiang contributed to data analysis, and text revision.

\section{References}

Bai Y, Yao L, Wei T, Tian F, Jin DY, Chen L, Wang M. Presumed Asymptomatic Carrier Transmission of COVID-19. JAMA 2020; 323: 1406-1407.

Baumgarth N. How specific is too specific? B-cell responses to viral infections reveal the importance of breadth over depth. Immunol Rev 2013; 255: 82-94.

Corman VM, Landt O, Kaiser M, Molenkamp R, Meijer A, Chu DK, Bleicker T, Brünink S, Schneider J, Schmidt ML, Mulders DG. Detection of 2019 novel coronavirus (2019-nCoV) by real-time RT-PCR. Euro Surveill 2020; 25: 2000045.

Duan K, Liu B, Li C, Zhang H, Yu T, Qu J, Zhou M, Chen L, Meng S, Hu Y, Peng C. Effectiveness of convalescent plasma therapy in severe COVID-19 patients. Proc Natl Acad Sci USA 2020; 117: 9490-9496.

Lam JH and Baumgarth N. The Multifaceted B Cell Response to Influenza Virus. J Immunol 2019; 202: 351-359.

Long QX, Liu BZ, Deng HJ, Wu GC, Deng K, Chen YK, Liao P, Qiu JF, Lin Y, Cai XF, Wang DQ. Antibody responses to SARSCoV-2 in patients with COVID-19. Nat Med 2020; 29: 1-4.

Rothe C, Schunk M, Sothmann P, Bretzel G, Froeschl G, Wallrauch C, Zimmer T, Thiel V, Janke C, Guggemos W, Seilmaier M. Transmission of 2019-nCoV Infection from an Asymptomatic Contact in Germany. N Engl J Med 2020; 382: 970-971.

Wang Y, Liu Y, Liu L, Wang X, Luo N, Ling L. Clinical outcome of 55 asymptomatic cases at the time of hospital admission infected with SARS-Coronavirus-2 in Shenzhen, China. J Infect Dis 2020.

Xiang F, Wang X, He X, Peng Z, Yang B, Zhang J, Zhou Q, Ye H, Ma Y, Li H, Wei X. Antibody Detection and Dynamic 
Yongchen Z, Shen H, Wang X, Shi X, Li Y, Yan J, Chen Y, Gu B. Different longitudinal patterns of nucleic acid and serology testing results based on disease severity of COVID-19 patients. Emerg Microbes Infect 2020; 9: 833-836.

Zhang J, Wu S, Xu L. Asymptomatic carriers of COVID-19 as a concern for disease prevention and control: more testing, more follow-up. Biosci Trends 2020.

Zhang L, Pang R, Xue X, Bao J, Ye S, Dai Y et al. Anti-SARS-CoV-2 virus antibody levels in convalescent plasma of six donors who have recovered from COVID-19. Aging (Albany NY) 2020; 12: 6536-6542.

Zhang L, Zhang F, Yu W, He T, Yu J, Yi CE, Ba L, Li W, Farzan M, Chen Z, Yuen KY. Antibody responses against SARS coronavirus are correlated with disease outcome of infected individuals. J Med Virol 2006; 78: 1-8.

Zhao J, Yuan Q, Wang H, Liu W, Liao X, Su Y, Wang X, Yuan J, Li T, Li J, Qian S. Antibody responses to SARS-CoV-2 in patients of novel coronavirus disease 2019. Clin Infect Dis 2020.

Zhu N, Zhang D, Wang W, Li X, Yang B, Song J, Zhao X, Huang B, Shi W, Lu R, Niu P. A Novel Coronavirus from Patients with Pneumonia in China, 2019. N Engl J Med 2020; 382: 727-733. 